\title{
Association of serum uric acid with clinical and radiological severity of knee osteoarthritis in non-gouty patients
}

\author{
Sherine Abdel Rahman Abdel Karim Bassiouni ${ }^{1 *} \mathbb{D}$, Mohamed Ali El Adalany², Maha Abdelsalam ${ }^{3,4}$ and \\ Ola Mohammad Gharbia ${ }^{1}$
}

\begin{abstract}
Background: A growing body of evidence suggested that uric acid (UA) may contribute in the pathways underlying osteoarthritis (OA) pathogenesis; however, studies that investigated the relationship between $\cup A$ and OA emerged inconclusive results. The purpose of the study was to explore the association of serum uric acid (sUA) levels with clinical severity, radiological severity of knee osteoarthritis (KOA) based on Kellgren-Lawrence (KL) grading system, and MRI changes in non-gouty patients.
\end{abstract}

Results: WOMAC scores: pain, stiffness, function, and total score are significantly higher in H-sUA group than L-sUA group ( $p=0.004, p=0.019, p=0.018, p=0.008$ respectively). Joint space width (JSW) is significantly narrower in H-sUA group than L-s $\cup A$ group $(p=0.013)$. H-s $\cup A$ group had more frequent $\mathrm{KL}$ grade $4(p<0.001)$, osteophytes grade 4 $(p<0.001)$, focal bone erosion $(p<0.001)$, bone marrow lesions $(p=0.023)$, and synovitis $(p=0.011)$ than $L-s \cup A$ group. Female KOA patients in H-sUA group had significantly higher pain, stiffness, and function and total WOMAC scores than L-sUA group ( $p=0.003, p=0.015, p=0.008, p=0.004)$, more frequently had $\mathrm{KL}$ grade 4 and osteophytes grade 4 $(p=0.003, p<0.001)$, significantly narrower JSW $(p=0.016)$, more frequently show focal bone erosion $(p=0.002)$, bone marrow lesions $(p=0.019)$, and synovitis $(p=0.004)$ than $L-s \cup A$ group. In regression analysis, female sex $(p=0.035)$, duration of KOA $(p=0.031)$, and sUA level $(p=0.025)$ were associated with KL severity. For female patients with KOA, KL severity is associated with duration of KOA $(p=0.045)$ and $\operatorname{SUA}(p=0.009)$.

Conclusion: Higher sUA level is associated with higher clinical severity, higher radiographic KL grades, and more frequent MRI findings in patients with primary KOA patients. Our results also indicated that sUA level was significantly associated with KOA severity in female patients, but not in male patients. More studies are warranted to explore whether the two conditions exist simultaneously or there is a direct causal relationship between the two conditions.

Keywords: Knee osteoarthritis, Serum uric acid, Joint space width, Osteophytes, Synovitis

\section{Background}

Osteoarthritis $(\mathrm{OA})$ is a leading cause of chronic pain, impaired mobility, and reduced quality of life in the elderly population [1]. Knee OA (KOA) is the 11th highest contributor to global disability [2] and accounts for $>$ $80 \%$ of the total burden of the diseases [3]. At least $19 \%$

\footnotetext{
* Correspondence: sherineabdelrahman@yahoo.com

'Physical Medicine, Rheumatology and Rehabilitation Department, Faculty of Medicine, Mansoura University, Mansoura, Egypt

Full list of author information is available at the end of the article
}

of adults aged 45 years or older had KOA [4]. The lifetime risk of developing symptomatic KOA is 45\% (40\% in males and $47 \%$ in females), with higher risk ( 60.5\%) in obese persons [5].

KOA results from a combination of breakdown of articular cartilage due mechanical loading [6] and chronic inflammation [7]; however, the deeper molecular mechanisms underlying KOA remain largely unclear and scantily investigated, hindering efforts to develop strategy for disease prevention and treatment [8]. On the other hand, 
KOA is typically a slowly progressing condition; however, some patients had a rapid progression of structural damage [9]. Therefore, advances in our understanding of KOA pathogenesis may lead to introduction of novel therapeutic strategies for this condition [10].

Uric acid (UA) is a metabolically active molecule. Urate deposition within joints and soft tissues can induce acute painful gouty arthritis. However, UA can also drive low-level chronic inflammatory state, even in the absence of gout [11, 12]. Epidemiologically, old age and obesity are common risk factors for OA and urate deposition in tissues and both conditions share a common tendency for certain joints, including the knee joint [10]. Additionally, OA is associated with interleukin-1 (IL-1) response [13], a key mediator of the inflammatory response to urate deposition in tissues [14].

A growing body of evidence suggested that UA may contribute in the pathways underlying OA pathogenesis [15]; however, studies that investigated the relationship between UA and OA emerged inconclusive results. In the one hand, OA severity was found to be correlated with serum UA (sUA) level in non-gouty patients $[16,17]$. On the other hand, it was reported that sUA level was not significantly associated with KOA, although the same study observed a trend toward such an association in female patients with KOA [18]. The aim of this study is to explore the association of sUA levels with clinical and radiological severity of KOA based on Kellgren-Lawrence (KL) grading system and MRI changes in non-gouty patients.

\section{Methods}

\section{Patients selection}

A total of 200 consecutive patients with KOA were invited to participate in this cross-sectional study. Patients were recruited from the Outpatient Clinics of Rheumatology and Rehabilitation Department between June 2018 and June 2020. For inclusion in the study, patients should meet the American College of Rheumatology radiological and clinical criteria for diagnosis of KOA [19].

We excluded patients with secondary KOA, history of gouty arthritis, patients with metabolic syndrome or any of its individual components, any other form of knee arthritis (including other crystal arthritis, infectious arthritis, inflammatory arthritis, or seronegative spondylo-arthropathy), previous knee surgery, or knee trauma, patients currently using UA-lowering medication, and hyaluronan and/or corticosteroid injection to the affected knee within 3 past months. Patients with body mass index $(\mathrm{BMI}) \geq 33 \mathrm{~kg} / \mathrm{m}^{2}$ were not enrolled in this study to minimize impact of obesity on OA/inflammatory biomarkers [20]. For the current study, we included only patients whose KOA was predominantly medial (i.e., medial joint space width (JSW) less than lateral JSW on radiographic assessment), consistent with the OA Research Society International recommendations [21].

Patients were informed about study aim and procedures prior to inclusion in the study. Patients willing to participate in the study had provided written informed consent. The study was approved by the local Ethical Committee.

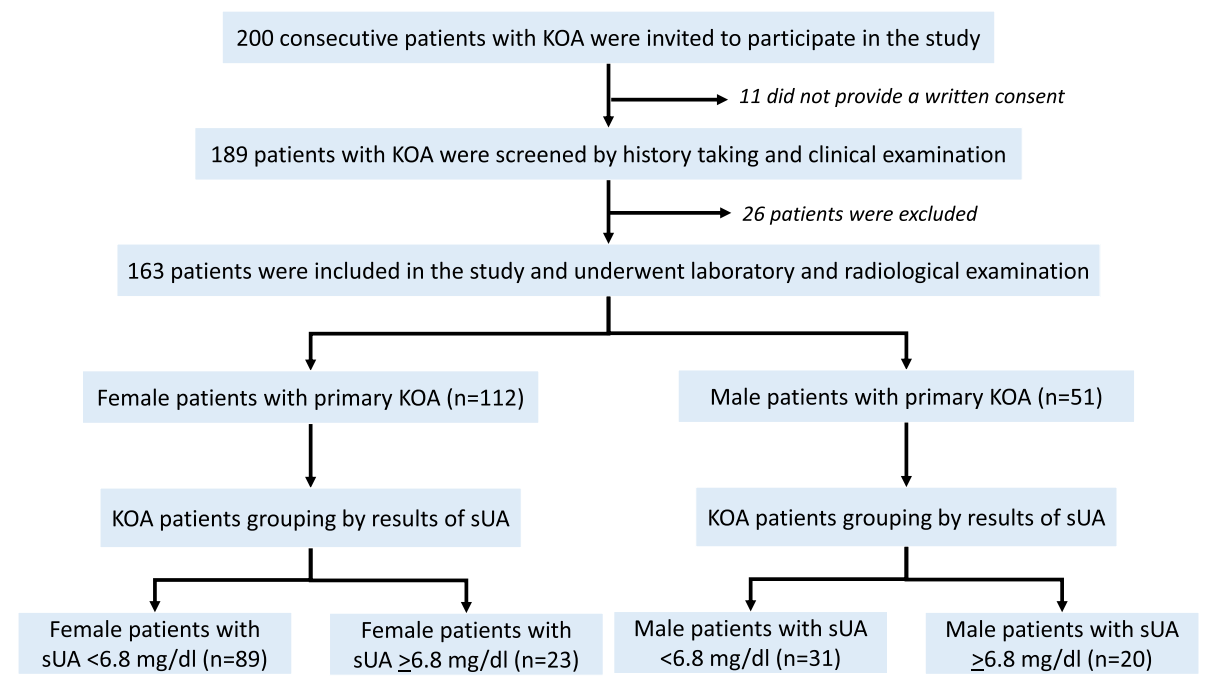

Fig. 1 Flowchart for KOA patients participated in the study 


\section{Clinical data and laboratory measures}

Patients were clinically assessed through full history taking followed by general and local examinations. Data regarding age, sex, duration of $\mathrm{KOA}$, presence of comorbidities, and current medications were recorded and BMI was calculated. All patients completed Western Ontario and McMaster Universities OA Index (WOMAC) questionnaire [22].

Venous blood sample was withdrawn from antecubital vein from every patient in the morning after twelve hours overnight fast. Serum samples had been collected in serum collection tubes, and serum has been isolated within $60 \mathrm{~min}$ of collection then placed in aliquots and stored at $-70{ }^{\circ} \mathrm{C}$ until time of assessment of sUA levels. The sUA measurements were performed at the Clinical Pathology labs by the automated colorimetric assay using a Cobas c 111 analyzer-Roche, Germany. The patients were subdivided into two groups according to the solubility point of sUA at body temperature $(=6.8 \mathrm{mg} /$ dl) [23]; a group of patients with low sUA $(<6.8 \mathrm{mg} / \mathrm{dl})$ "L-sUA group" and the other group included the patients with high sUA ( $\geq 6.8 \mathrm{mg} / \mathrm{dl})$ "High-sUA group."

\section{Radiological examination}

All radiological images were performed and interpreted by the same experienced musculoskeletal radiologist

Table 1 Comparison of socio-demographic characteristics, clinical findings, and radiological findings between knee osteoarthritis patients in low serum uric acid group versus high serum uric acid group

\begin{tabular}{|c|c|c|c|}
\hline & L-sUA group & H-sUA group & $P$ \\
\hline Age (years) (mean \pm SD) & $59.7 \pm 5.8$ & $59.5 \pm 6.8$ & $0.898^{*}$ \\
\hline Females sex (, \%) & $89,74.2 \%$ & $23,53.5 \%$ & $0.012^{* *}$ \\
\hline \multicolumn{4}{|l|}{ Residence $(n, \%)$} \\
\hline Rural & $54,45.0 \%$ & $20,46.5 \%$ & \\
\hline Urban & $66,55.0 \%$ & $23,53.5 \%$ & $0.864^{* *}$ \\
\hline \multicolumn{4}{|l|}{ Educational level $(n, \%)$} \\
\hline Basic & $36,30.0 \%$ & $11,25.6 \%$ & \\
\hline Secondary & $34,28.3 \%$ & $11,25.6 \%$ & \\
\hline Higher & $50,41.7 \%$ & $21,48.8 \%$ & $0.714^{* *}$ \\
\hline $\mathrm{BMI}\left(\mathrm{kg} / \mathrm{m}^{2}\right)($ mean $\pm \mathrm{SD})$ & $22.6 \pm 1.4$ & $22.8 \pm 1.6$ & $0.452^{*}$ \\
\hline Duration of KOA (years) (mean $\pm S D$ ) & $10.7 \pm 3.2$ & $10.3 \pm 3.7$ & $0.469^{*}$ \\
\hline \multicolumn{4}{|l|}{ WOMAC score $($ mean $\pm S D)$} \\
\hline Pain subscale & $8.3 \pm 3.6$ & $10.2 \pm 4.0$ & $0.004^{*}$ \\
\hline Stiffness subscale & $2.2 \pm 1.1$ & $2.6 \pm 1.2$ & $0.019^{*}$ \\
\hline Function subscale & $30.8 \pm 9.8$ & $35.0 \pm 10.1$ & $0.018^{*}$ \\
\hline Total score & $41.2 \pm 13.7$ & $47.8 \pm 14.1$ & $0.008^{*}$ \\
\hline \multicolumn{4}{|l|}{$\mathrm{KL}$ grade $(n, \%)$} \\
\hline Grade 2 & $58,48.3 \%$ & $10,23.3 \%$ & \\
\hline Grade 3 & $43,35.8 \%$ & $15,34.9 \%$ & \\
\hline Grade 4 & $19,15.8 \%$ & $18,41.9 \%$ & $<0.001^{* *}$ \\
\hline \multicolumn{4}{|l|}{ Osteophytes (n, \%) } \\
\hline Grade 2 & $60,50.0 \%$ & $8,18.6 \%$ & \\
\hline Grade 3 & $43,35.8 \%$ & $14,32.6 \%$ & \\
\hline Grade 4 & $17,14.2 \%$ & $21,48.8 \%$ & $<0.001^{* *}$ \\
\hline JSW (mm) (median [IQR]) & $1.75[1.67]$ & $1.10[1.70]$ & $0.013^{* * *}$ \\
\hline Focal erosion $(n, \%)$ & $47,39.2 \%$ & $30,69.8 \%$ & $<0.001^{* *}$ \\
\hline Bone marrow lesions $(n, \%)$ & $38,31.7 \%$ & $22,51.2 \%$ & $0.023^{* *}$ \\
\hline Effusion $(n, \%)$ & $48,40.0 \%$ & $18,41.9 \%$ & $0.831^{* *}$ \\
\hline Synovitis ( $n, \%)$ & $43,35.8 \%$ & $25,58.1 \%$ & $0.011^{* *}$ \\
\hline Soft tissue swelling $(n, \%)$ & $40,33.3 \%$ & $18,41.9 \%$ & $0.316^{*}$ \\
\hline
\end{tabular}

KOA knee OA, L-sUA low serum uric acid, H-sUA high serum uric acid, SD standard deviation, BMI body mass index, WOMAC Western Ontario and McMaster Universities OA Index, KL Kellgren-Lawrence, JSW joint space width, $\mathrm{mm}$ millimeters, IQR interquartile range

* Student's $t$ test, ${ }^{* *}$ chi-square test, ${ }^{* * *}$ Mann-Whitney $U$ test, significance level was set at $p \leq 0.05$ 
who was blinded to the clinical and laboratory findings of the patients.

Patients underwent $45^{\circ}$ posteroanterior flexion weightbearing plain radiograph of both knee joints. The KOA severity and osteophytes grading was determined according to the Kellgren-Lawrence (KL) grading system [24]. The minimum JSW, defined as the inter-bone distance, was measured in millimeters $(\mathrm{mm})$ at the medial tibiofemoral joint space [25]. The knee joint with higher $\mathrm{KL}$ grade was recognized as the index knee. Only the data of the index were analyzed in this study. For magnetic resonance image (MRI) examination, patients were placed in supine position with the knee joint extended. MRI inflammatory and structural changes including synovitis, focal bone erosions, effusion, bone marrow lesion, and soft tissue swelling were reported as present or absent.

\section{Statistical analysis}

Continuous variables were normally distributed and were presented as mean \pm standard deviation (SD). Categorical data were presented in number and percentage. Comparisons were determined by using independent sample Student's $t$ test for two variables with continuous data. Chi-square test was used for comparison of variables with categorical data. Multivariable-adjusted association between radiographic severity of $\mathrm{KOA}$, measured by $\mathrm{KL}$ grade and the potential covariant variables was evaluated by logistic regression in patients with $\mathrm{KOA}$ and in female patients with KOA. All data analyses were performed using SPSS version 20.0. $P \leq 0.05$ was considered statistically significant.

\section{Results}

A total of 200 consecutive patients with KOA were invited to participate in the study. Among these patients, 11 did not provide a written consent for participation in the study and were excluded from the study. Another 26 patients were excluded from the study due to BMI $>33$ $\mathrm{kg} / \mathrm{m}^{2}(n=8)$, hyaluronan injection $(n=8)$, or corticosteroid injection $(n=3)$ of the affected knee within 3 months, secondary KOA $(n=5)$ and previous trauma/ surgery $(n=2)$, leaving 163 patients, 112 females and 51 males, eligible to participate in the study. The average level of sUA in the eligible KOA patients was $5.6 \pm 1.2$ $\mathrm{mg} / \mathrm{dl}$. The flowchart for patient selection and grouping is demonstrated in Fig. 1.

\section{KOA patients}

As shown in Table 1, the WOMAC pain, stiffness, and function scores are significantly higher in KOA patients in the $\mathrm{H}$-sUA group compared to L-sUA group ( $p=$ $0.004, p=0.019$ and $p=0.018$ respectively). The total WOMAC score was also significantly higher in KOA patients with $\mathrm{H}$-sUA than L-sUA $(p=0.008)$.

On plain radiography imaging, $\mathrm{H}$-sUA group showed more severe changes than L-sUA group of patients. The $\mathrm{KL}$ grade 4 in plain radiography is more frequent in the H-sUA group than L-sUA group ( $41.9 \%$ vs. $15.8 \%$ respectively, $p<0.001)$. The osteophytes in grade 4 were also more frequently seen in the $\mathrm{H}$-sUA group than $\mathrm{L}$ -

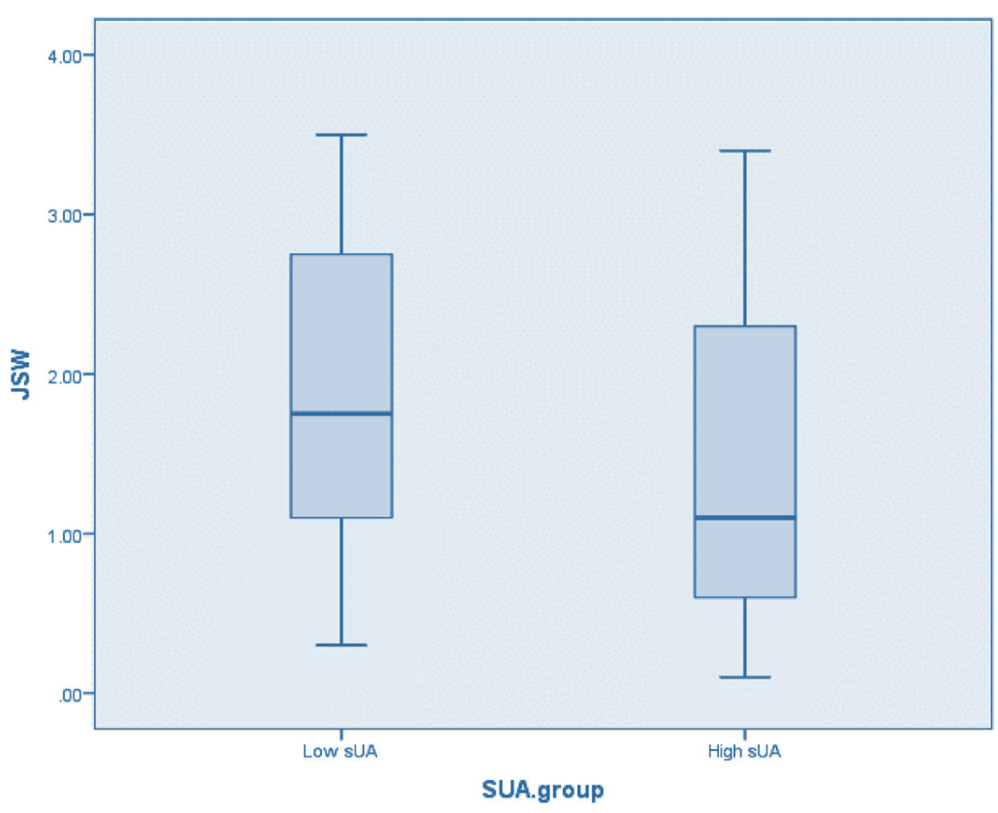

Fig. 2 Comparison of the JSW between KOA patients in L-sUA group versus $\mathrm{H}$-s $U A$ group 
sUA group ( $48.8 \%$ vs. $14.2 \%$ respectively, $p<0.001)$ (Table 1). In addition, the JSW is significantly narrower in the H-sUA group than L-sUA group $(p=0.013)$ (Table 1, Fig. 2).

On MRI examination, patients in $\mathrm{H}$-sUA group more frequently show focal bone erosion $(69.8 \%$ vs. $39.2 \%$ respectively), bone marrow lesions $(51.2 \%$ vs. $31.7 \%$ respectively), and synovitis (58.1\% vs. $35.8 \%$ respectively) than L-sUA group. These differences were significant ( $p$ $<0.001, p=0.023$, and $p=0.011$ respectively) (Table 1 ).

\section{Female KOA patients}

Females KOA patients in H-sUA group had significantly higher pain, stiffness, and function scores than L-sUA group ( $p=0.003, p=0.015, p=0.008$ respectively). The WOMAC total score was also significantly higher in $\mathrm{H}$ sUA than L-sUA group of females with $\mathrm{KOA}(p=$ 0.004). Female patients with KOA in H-sUA group more frequently had KL grade 4 (39.1\% vs. $11.2 \%$ respectively) and osteophytes grade 4 ( $56.5 \%$ and $11.2 \%$ respectively). These differences were significant $(p=0.003$ and $p<$ 0.001 respectively) (Table 2). Moreover, JSW is significantly narrower in female KOA patients in $\mathrm{H}$-sUA group than L-sUA group ( $p=0.016)$ (Table 2, Fig. 3).

On MRI examination, female patients in H-sUA group more frequently show focal bone erosion $(73.9 \%$ vs. $37.1 \%$ respectively), bone marrow lesions $(56.5 \%$ vs. $30.3 \%$ respectively), and synovitis $(73.9 \%$ vs. $40.4 \%$

Table 2 Comparison of socio-demographic characteristics, clinical findings, and radiological findings between female knee osteoarthritis patients in low serum uric acid group versus high serum uric acid group

\begin{tabular}{|c|c|c|c|}
\hline & L-sUA group & H-sUA group & $P$ \\
\hline Age (years) (mean \pm SD) & $59.4 \pm 5.6$ & $59.5 \pm 6.7$ & $0.970^{*}$ \\
\hline \multicolumn{4}{|l|}{ Residence $(n, \%)$} \\
\hline Rural & $37,41.6 \%$ & $10,43.5 \%$ & \\
\hline Urban & $52,58.4 \%$ & $13,56.5 \%$ & $0.869^{* *}$ \\
\hline \multicolumn{4}{|l|}{ Educational level $(n, \%)$} \\
\hline Basic & $28,31.5 \%$ & $5,21.7 \%$ & \\
\hline Secondary & $25,28.1 \%$ & $6,26.1 \%$ & \\
\hline Higher & $36,40.4 \%$ & $12,52.2 \%$ & $0.549^{* *}$ \\
\hline $\mathrm{BMI}\left(\mathrm{kg} / \mathrm{m}^{2}\right)($ mean $\pm \mathrm{SD})$ & $22.6 \pm 1.4$ & $22.7 \pm 1.7$ & $0.790^{*}$ \\
\hline Duration of KOA (years) (mean $\pm S D$ ) & $10.9 \pm 3.1$ & $10.7 \pm 3.7$ & $0.732^{*}$ \\
\hline \multicolumn{4}{|l|}{ WOMAC score (mean \pm SD) } \\
\hline Pain subscale & $8.4 \pm 3.5$ & $10.9 \pm 4.0$ & $0.003^{*}$ \\
\hline Stiffness subscale & $2.1 \pm 1.0$ & $2.7 \pm 1.3$ & $0.015^{*}$ \\
\hline Function subscale & $30.7 \pm 9.8$ & $36.9 \pm 9.6$ & $0.008^{*}$ \\
\hline Total score & $41.2 \pm 13.6$ & $50.5 \pm 13.6$ & $0.004^{*}$ \\
\hline \multicolumn{4}{|l|}{$\mathrm{KL}$ grade $(n, \%)$} \\
\hline Grade 2 & $48,53.9 \%$ & $6,26.1 \%$ & \\
\hline Grade 3 & $31,34.8 \%$ & $8,34.8 \%$ & \\
\hline Grade 4 & $10,11.2 \%$ & $9,39.1 \%$ & $0.003^{* *}$ \\
\hline \multicolumn{4}{|l|}{ Osteophytes $(n, \%)$} \\
\hline Grade 2 & $45,50.6 \%$ & $3,13.0 \%$ & \\
\hline Grade 3 & $35,39.3 \%$ & $7,30.4 \%$ & \\
\hline Grade 4 & $9,10.1 \%$ & $13,56.5 \%$ & $<0.001^{* *}$ \\
\hline JSW (mm) (median [IQR]) & $2.40[1.60]$ & $1.30[2.10]$ & $0.016^{* * *}$ \\
\hline Focal erosion $(n, \%)$ & $33,37.1 \%$ & $17,73.9 \%$ & $0.002^{* *}$ \\
\hline Bone marrow lesions $(n, \%)$ & $27,30.3 \%$ & $13,56.5 \%$ & $0.019^{* *}$ \\
\hline Effusion ( $n, \%)$ & $28,31.5 \%$ & $8,34.8 \%$ & $0.761^{* *}$ \\
\hline Synovitis $(n, \%)$ & $36,40.4 \%$ & $17,73.9 \%$ & $0.004^{* *}$ \\
\hline Soft tissue swelling $(n, \%)$ & $28,31.5 \%$ & $9,39.1 \%$ & $0.486^{* *}$ \\
\hline
\end{tabular}

KOA knee OA, L-sUA low serum uric acid, H-sUA high serum uric acid, SD standard deviation, BMI body mass index, WOMAC Western Ontario and McMaster Universities OA Index, KL Kellgren-Lawrence, JSW joint space width, $\mathrm{mm}$ millimeters, IQR interquartile range

* Student's $t$ test, ${ }^{* *}$ chi-square test, ${ }^{* * *}$ Mann-Whitney $U$ test, significance level was set at $p \leq 0.05$ 


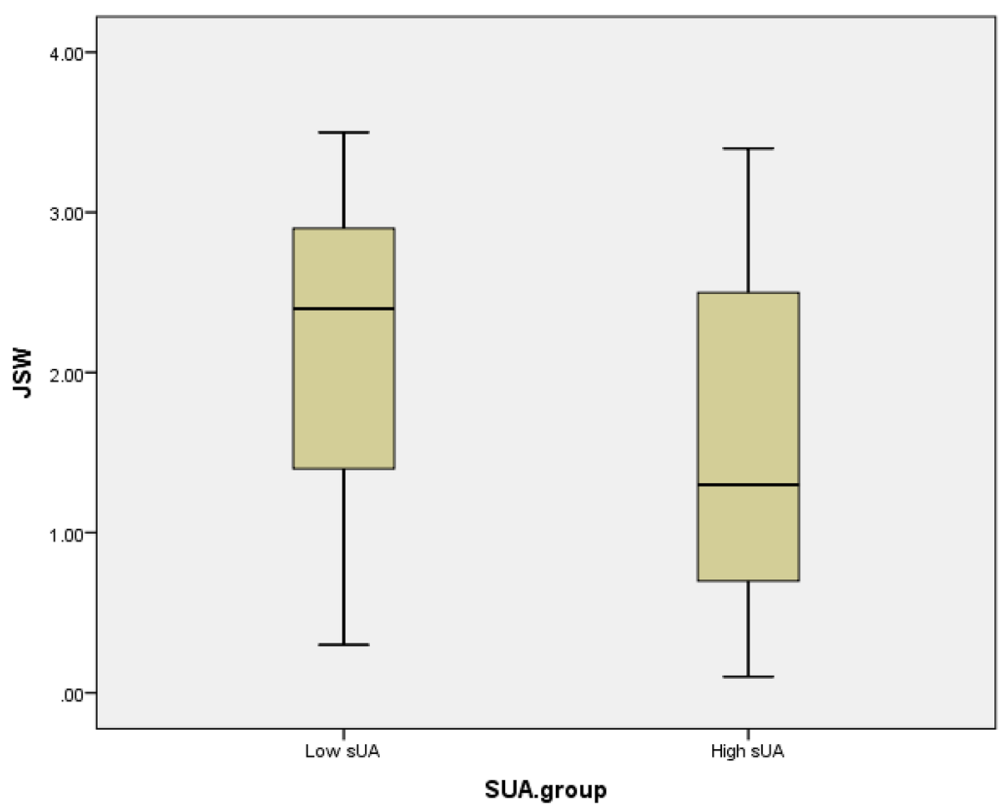

Fig. 3 Comparison of the JSW between female KOA patients in L-sUA group versus $\mathrm{H}$-sUA group

respectively) than patients in L-sUA group. These differences were significant $(p=0.002, p=0.019, p=0.004$ respectively) (Table 2).

\section{Male KOA patients}

No significant difference was found as regards the clinical or imaging findings between the male KOA patients in $\mathrm{H}$ sUA group in comparison to L-sUA group (Table 3).

\section{Regression analysis}

The multivariable-adjusted associations with the severity of KOA measured by the KL grade in patients with $\mathrm{KOA}$ and in female patients with KOA are shown in Tables 4 and 5. Factors that demonstrated significant association with the KL grade in patients with $\mathrm{KOA}$ include female sex $(p=0.035)$, duration of KOA $(p=0.031)$, and sUA level $(p=0.025)$. For female patients with KOA, a significant association between KL grade and duration of KOA $(p=0.045)$ and sUA $(p=0.009)$ was observed.

\section{Discussion}

The results of the present study revealed that KOA patients in H-sUA group had significantly worse WOMAC pain, stiffness, and function scores and worse WOMAC total score than patients in L-sUA group. In addition, $\mathrm{H}$ sUA group showed more severe $\mathrm{x}$-ray changes, as they display significantly more frequent KL grade 4 , more frequent grade 4 osteophytes, and narrower JSW than LsUA group. On MRI examination, patients in H-sUA group more frequently show focal bone erosion, bone marrow lesions and synovitis than patients in L-sUA group.
Xiao et al. [17] enrolled 71 patients with primary KOA without previous attack of gout, and patients were categorized into two groups based on the level of sUA at a cutoff point of $360 \mu \mathrm{mol} / \mathrm{L}$ (equivalent to $6.05 \mathrm{mg} / \mathrm{dl}$ ). The aim of that study was to assess the association between sUA and MRI-based assessment of KOA severity. The study found that KOA patients with sUA $>360$ $\mu \mathrm{mol} / \mathrm{L}$ had significantly more focal erosions, osteophytes, bone marrow lesions, and synovitis and soft tissue swelling than patients with sUA $<360 \mu \mathrm{mol} / \mathrm{L}$. The study concluded that primary KOA patients with higher sUA level were more likely to have more severe MRI findings, in agreement with our findings.

In the cross-sectional study of Srivastava et al. [26], that included 570 patients with primary KOA without history of gout, the results revealed that the patients with higher sUA had significantly more severe WOMAC score of the three subscales; pain, stiffness, and function as well as total WOMAC score. In addition, the study reported a significant increase in sUA levels with radiological severity in terms of KL grade.

Also, in support with our findings, previous studies had found that the highest tertile of sUA level is associated with osteophytes in KOA [27] and even with generalized OA [28]. In addition, one previous study that explored the relationship of sUA and OA gave the conclusion that KOA prevalence and severity were significantly increased in patients with elevated sUA [29].

In contrast, Krasnokutsky et al. [20] found that sUA did not correlate with KOA severity measured as WOMAC pain score, KL grade, and JSW. In that study, $22.7 \%$ of the KOA patients had KL grade 1 instead of 
Table 3 Comparison of socio-demographic characteristics, clinical findings, and radiological findings between male knee osteoarthritis patients in low serum uric acid group versus high serum uric acid group

\begin{tabular}{|c|c|c|c|}
\hline & L-sUA group & H-sUA group & $p$ \\
\hline Age (years) (mean $\pm S D$ ) & $60.3 \pm 6.5$ & $59.6 \pm 7.1$ & $0.703^{*}$ \\
\hline \multicolumn{4}{|l|}{ Residence } \\
\hline Rural & $17,54.8 \%$ & $10,50.0 \%$ & \\
\hline Urban & $14,45.2 \%$ & $10,50.0 \%$ & $0.735^{* *}$ \\
\hline \multicolumn{4}{|l|}{ Educational level $(n, \%)$} \\
\hline Basic & $8,25.8 \%$ & $6,30.0 \%$ & \\
\hline Secondary & $9,29.0 \%$ & $5,25.0 \%$ & \\
\hline Higher & $14,45.2 \%$ & $9,45.0 \%$ & $0.928^{* *}$ \\
\hline $\mathrm{BMI}\left(\mathrm{kg} / \mathrm{m}^{2}\right)($ mean $\pm \mathrm{SD})$ & $22.7 \pm 1.6$ & $23.0 \pm 1.6$ & $0.512^{*}$ \\
\hline Duration of KOA (years) & $10.1 \pm 3.5$ & $9.9 \pm 3.7$ & $0.787^{*}$ \\
\hline \multicolumn{4}{|l|}{ WOMAC score (mean \pm SD) } \\
\hline Pain subscale & $8.1 \pm 3.9$ & $9.4 \pm 4.0$ & $0.242^{*}$ \\
\hline Stiffness subscale & $2.3 \pm 1.2$ & $2.5 \pm 1.1$ & $0.542^{*}$ \\
\hline Function subscale & $30.8 \pm 9.8$ & $32.8 \pm 10.5$ & $0.512^{*}$ \\
\hline Total score & $41.2 \pm 14.2$ & $44.7 \pm 14.4$ & $0.403^{*}$ \\
\hline \multicolumn{4}{|l|}{$\mathrm{KL}$ grade $(n, \%)$} \\
\hline Grade 2 & $10,32.3 \%$ & $4,20.0 \%$ & \\
\hline Grade 3 & $12,38.7 \%$ & $7,35.0 \%$ & \\
\hline Grade 4 & $9,29.0 \%$ & $9,45.0 \%$ & $0.452^{* *}$ \\
\hline \multicolumn{4}{|l|}{ Osteophytes ( $n, \%)$} \\
\hline Grade 2 & $15,48.4 \%$ & $5,25.0 \%$ & \\
\hline Grade 3 & $8,25.8 \%$ & $7,35.0 \%$ & \\
\hline Grade 4 & $8,25.8 \%$ & $8,40.0 \%$ & $0.243^{* *}$ \\
\hline JSW (mm) (median [IQR]) & $1.10[0.80]$ & $1.05[1.73]$ & $0.575^{* * *}$ \\
\hline Focal erosion $(n, \%)$ & $14,45.2 \%$ & $13,65.0 \%$ & $0.166^{* *}$ \\
\hline Bone marrow lesions $(n, \%)$ & $11,35.5 \%$ & $9,45.0 \%$ & $0.497^{* *}$ \\
\hline Effusion ( $n, \%)$ & $20,64.5 \%$ & $10,50.0 \%$ & $0.304^{* *}$ \\
\hline Synovitis $(n, \%)$ & $7,22.6 \%$ & $8,40.0 \%$ & $0.183^{* *}$ \\
\hline Soft tissue swelling ( $n, \%)$ & $12,38.7 \%$ & $9,45.0 \%$ & $0.656^{* *}$ \\
\hline
\end{tabular}

KOA knee OA, L-sUA low serum uric acid, $H$-sUA high serum uric acid, $S D$ standard deviation, $B M I$ body mass index, WOMAC Western Ontario and McMaster Universities OA Index, KL Kellgren-Lawrence, JSW joint space width, $\mathrm{mm}$ millimeters, $I Q R$ interquartile range

* Student's $t$ test, ${ }^{* *}$ chi-square test, ${ }^{* * *}$ Mann-Whitney $U$ test, significance level was set at $p \leq 0.05$

the more conventionally defined KL score $\geq 2$ for defining OA used in the present study which may explain the inconsistency between the findings of the present study from that of Krasnokutsky et al. However, the same study reported that $\mathrm{KOA}$ patients with sUA level $\geq 6.8$ $\mathrm{mg} / \mathrm{dl}$ at baseline had significantly narrower JSW and more evident synovitis after 24 months than KOA patients with sUA level $<6.8 \mathrm{mg} / \mathrm{dl}$ in the univariate analysis and the correlation remained significant in the multivariate analysis. This later finding provides a clue for the role of sUA in the progression of severity of the KOA.

In support to the relationship between the sUA and WOMAC score, two randomized clinical trials showed a significant improvement rate of total WOMAC score from 57.9 to $23.5 \%$ [30] and from 49 to $39 \%$ [31] at 20 weeks in a group of patients receiving colchicine than in control group receiving placebo.

Next, we categorized the patients that participated in this study into two gender-based subgroups to determine whether gender contributes to the harmful effects of sUA on KOA. Our results showed that female patients with $\mathrm{KOA}$ in $\mathrm{H}$-sUA group had significantly more severe WOMAC subscales and total scores, and in plain $\mathrm{x}$-ray, had more frequent $\mathrm{KL}$ grade 4 , more frequent grade 4 osteophytes, and narrower JSW than female patients in L-sUA group. On MRI examination, female patients with $\mathrm{KOA}$ in $\mathrm{H}$-sUA group more frequently showed focal bone erosion, bone marrow lesions, and synovitis than patients in L-sUA group. In contrast, there were no such significant differences regarding clinical and radiological findings between male patients with $\mathrm{KOA}$ in $\mathrm{H}$-sUA group as compared to L-sUA group.

In agreement with our findings, a Chinese study that assessed the association between sUA and radiographic changes in 4685 OA patients revealed that the highest tertile of sUA level was positively correlated with osteophytes in female patients with KOA, but not in male patients [27]. In support for the gender differences regarding the effect of sUA, previous studies have observed that the sUA level was related to KOA and also with hand $\mathrm{OA}$ in female patients, but not in male patients [32, 33]. The findings obtained by Kim et al.'s [18] study indicated that sUA level was not significantly associated with $\mathrm{OA}$ in the male patients with OA, although there was a trend toward such a relationship in female patients. The gender differences regarding the association of sUA with KOA severity can be attributed to the abnormal biomechanical environments and aberrant sex hormone modulation as it had been shown that sUA rise significantly after menopause [34, 35].

To determine the factors related to severity of KOA, based on KL grade, we performed regression analysis in patients with KOA and in the subgroup of females with KOA. In regression analysis, female sex, duration of $\mathrm{KOA}$, and sUA level were associated with $\mathrm{KL}$ grade in KOA patients. For female patients with KOA, a significant association between $\mathrm{KL}$ grade and duration of $\mathrm{KOA}$ and sUA was observed. Consistent to our findings, Ding et al. [27] found a positive association between osteophyte severity and sUA in female patients in the multivariate analysis and the prevalence of osteophytes was increased in the highest tertile of UA compared to the 
Table 4 Multivariate regression analysis for factors associated with Kellgren-Lawrence grade in patients with knee osteoarthritis

\begin{tabular}{|c|c|c|c|c|c|}
\hline & \multicolumn{2}{|c|}{ Unstandardized coefficients } & \multirow{2}{*}{$\begin{array}{l}\text { Standardized Coefficients } \\
\text { Beta }\end{array}$} & \multirow[t]{2}{*}{$t$} & \multirow[t]{2}{*}{$p$} \\
\hline & $\bar{B}$ & Std. error & & & \\
\hline \multicolumn{6}{|c|}{ In KOA patients } \\
\hline (Constant) & -5.767 & 2.934 & & -1.965 & 0.052 \\
\hline Age & -0.261 & 0.137 & -0.173 & -1.902 & 0.060 \\
\hline Sex & -0.284 & 0.133 & -1.216 & -2.134 & 0.035 \\
\hline Residence & 0.230 & 0.139 & 0.153 & 1.656 & 0.101 \\
\hline Education & -0.075 & 0.081 & -0.084 & -0.924 & 0.358 \\
\hline BMI & 0.036 & 0.048 & 0.068 & 0.749 & 0.456 \\
\hline Duration & 0.136 & 0.062 & 0.203 & 2.189 & 0.031 \\
\hline SUA & 0.166 & 0.073 & 1.291 & 2.270 & 0.025 \\
\hline
\end{tabular}

KL Kellgren-Lawrence, KOA Knee OA, BMI Body mass index, SUA Serum uric acid

lowest among the female patients. These associations were not demonstrated in males.

Synovitis was thought to be a significant predictor for radiographic progression of hand OA including development of erosions [36], and MRI-detected synovitis is considered a risk factor for initiation and progression of OA $[37,38]$. Furthermore, synovitis in OA patients correlates with radiographic OA severity [39]. Our results revealed that synovitis was more frequent among KOA patient in H-sUA group compared to patients in L-sUA group, in agreement with previous studies $[17,20]$. The presence of significantly more frequent synovitis in KOA patients with higher sUA level provide an explanation why these patients demonstrated a more rapid and severe radiographic progression.

It had been suggested that synovial fluid is mainly a hyper-filtrate of serum. In this context, synovial membrane in OA may contribute to elevated urate level in the joint because OA synovium is permeable to water more than to urates and, hence, resorption of water from the joint space to circulation is faster than urate resorption in the resting joint leading to a relative elevation in urate concentration $[10,16]$.

The assumption that synovial fluid is mainly an ultrafiltrate of serum comes in support for the causal relationship between sUA levels and OA, with increased concentration of synovial fluid urate is promotor for damage of the cartilage [16]. Chondrocytes express urate transporters, and thus they are capable of taking up soluble urate. Urate deposition drives intra-cellular prooxidant effect and production of IL-1 $\beta$, a major cytokine involved in mechanisms underlying OA progression $[14]$. On the other hand, OA progression can be hastened intrinsically by chondrocytes death by UAdependent cycle [40]. Dying chondrocytes can locally generate UA, which act as a danger signal to activate inflammatory responses of neighboring cells promoting the pathological process of OA, which may explain why higher sUA levels were related to synovitis [41].

The present study had some limitations. The study included 163 patients with primary KOA. The relatively small sample size may increase the probability of type II

Table 5 Multivariate regression analysis for factors associated with Kellgren-Lawrence grade in female patients with knee osteoarthritis

\begin{tabular}{|c|c|c|c|c|c|}
\hline & \multicolumn{2}{|c|}{ Unstandardized coefficients } & \multirow{2}{*}{$\begin{array}{l}\text { Standardized Coefficients } \\
\text { Beta }\end{array}$} & \multirow[t]{2}{*}{$t$} & \multirow[t]{2}{*}{$p$} \\
\hline & B & Std. error & & & \\
\hline \multicolumn{6}{|c|}{ In female KOA patients } \\
\hline (Constant) & -0.241 & 1.850 & & -0.130 & 0.897 \\
\hline Age & 0.262 & 0.141 & 0.174 & 1.851 & 0.067 \\
\hline Residence & 0.015 & 0.041 & 0.118 & 0.369 & 0.713 \\
\hline Education & -0.084 & 0.083 & -0.095 & -1.018 & 0.311 \\
\hline BMI & 0.047 & 0.049 & 0.090 & 0.956 & 0.341 \\
\hline Duration & -0.284 & 0.140 & -0.189 & -2.030 & 0.045 \\
\hline SUA & 0.166 & 0.062 & 0.248 & 2.677 & 0.009 \\
\hline
\end{tabular}

KL Kellgren-Lawrence, KOA Knee OA, BMI Body mass index, SUA Serum uric acid 
error. Hence, more studies on larger sample sizes are necessary to confirm generalizability of the findings obtained by the present study. Elevated sUA levels are regarded as a component of metabolic syndrome and hence abdominal obesity is likely to be more relevant to be associated with KOA and hyperuricemia rather than BMI. Abdominal obesity and hyperuricemia are strongly associated with sedentary behavior and abdominal obesity. Exploring of the possible link between other confounding factors including the abdominal obesity is warranted. In the present study, we had classified our patients according to the solubility point of sUA and accordingly, at this cutoff point sUA level was significantly associated with KOA severity in female patients, but not in male patients probably suggesting that males may had a different cutoff point; this is an area of future study. In addition, a follow up study is warranted to explore the ability of the sUA to predict future deterioration of the osteoarthritic joint. The possible ability of sUA lowering drugs in hindering KOA progression had to be explored.

\section{Conclusion}

Higher sUA level is associated with higher clinical severity, higher radiographic KL grades, and more frequent MRI findings in patients with primary KOA patients. Our results also indicated that sUA level was significantly associated with KOA severity in female patients, but not in male patients. More studies are warranted to explore whether the two conditions exist simultaneously or there is a direct causal relationship between the two conditions.

\section{Abbreviations}

BMI: Body mass index; H-sUA: High serum uric acid; IL-1: Interleukin-1; JSW: Medial joint space width; KL: Kellgren-Lawrence; KOA: Knee OA; LsUA: Low serum uric acid; mm: Millimeters; MRI: Magnetic resonance image; OA: Osteoarthritis; SD: Standard deviation; sUA: Serum UA; UA: Uric acid; WOMAC: Western Ontario and McMaster Universities OA Index

\section{Acknowledgements}

Not applicable.

\begin{abstract}
Authors' contributions
Study conception and design, data acquisition and analysis, statistical analysis, drafting, and revising the manuscript by SA and OG. MA was responsible for study design, performing the radiological investigations, drafting, and revising the manuscript. MAS was responsible for study design, doing the laboratory investigations, drafting, and revising the manuscript. All authors read and approved the final manuscript.
\end{abstract}

\section{Funding}

No funding.

\section{Availability of data and materials}

The datasets used and/or analyzed during the current study are available from the corresponding author on reasonable request.

\section{Ethics approval and consent to participate}

The research was approved by the Institutional Review Board (IRB)/Mansoura Faculty of Medicine/Mansoura University (R/20.03.767). The written consent was obtained from all participants and approved by IRB.
Consent for publication

Not applicable in this study as there are no personal details.

\section{Competing interests}

The authors declare that they have no competing interests.

\section{Author details}

${ }^{1}$ Physical Medicine, Rheumatology and Rehabilitation Department, Faculty of Medicine, Mansoura University, Mansoura, Egypt. ${ }^{2}$ Diagnostic Radiology Department, Faculty of Medicine, Mansoura University, Mansoura, Egypt. ${ }^{3}$ Immunology Unit, Clinical Pathology Department, Faculty of Medicine, Mansoura University, Mansoura, Egypt. ${ }^{4}$ Immunology Department, Egypt Center for Research and Regenerative Medicine (ECRRM), Cairo 11517, Egypt.

Received: 6 November 2020 Accepted: 23 December 2020

Published online: 08 February 2021

\section{References}

1. Murray CJ, Atkinson C, Bhalla K, Birbeck G, Burstein R, Chou D et al (2013) The state of US health, 1990-2010: Burden of diseases, injuries, and risk factors. JAMA 310:591-608

2. Hoy DG, Smith E, Cross M, Sanchez-Riera L, Buchbinder R, Blyth FM et al (2014) The global burden of musculoskeletal conditions for 2010: an overview of methods. Ann Rheum Dis 73(6):982-989

3. Vos T, Flaxman AD, Naghavi M, Lozano R, Michaud C, Ezzati M et al (2012) Years lived with disability (YLDs) for 1160 sequelae of 289 diseases and injuries 1990-2010: A systematic analysis for the Global Burden of Disease Study 2010. Lancet 380:2163-2196

4. Lawrence RC, Felson DT, Helmick CG, Arnold LM, Choi H, Deyo RA et al (2008) Estimates of the prevalence of arthritis and other rheumatic conditions in the United States. Part II. Arthritis Rheum 58:26-35

5. Neogi T (2013) The epidemiology and impact of pain in osteoarthritis. Osteoarthritis Cartilage 21(9):1145-1153

6. Felson DT (2013) Osteoarthritis as a disease of mechanics. Osteoarthritis Cartilage 21(1):10-15

7. Robinson WH, Lepus CM, Wang Q, Raghu H, Mao R, Lindstrom TM et al (2016) Low-grade inflammation as a key mediator of the pathogenesis of osteoarthritis. Nat Rev Rheumatol 12(10):580-592

8. Wallace IJ, Worthington S, Felson DT, Jurmain RD, Wren KT, Maijanen $\mathrm{H}$ et al (2017) Knee osteoarthritis has doubled in prevalence since the mid-20th century. Proc Natl Acad Sci U S A 114(35):9332-9336

9. Driban JB, Eaton CB, Lo GH, Ward RJ, Lu B, MCAlindon TE (2014) Association of knee injuries with accelerated knee osteoarthritis progression: data from the Osteoarthritis Initiative. Arthritis Care Res 66(11):1673-1679

10. Neogi T, Krasnokutsky S, Pillinger MH (2019) Urate and osteoarthritis: evidence for a reciprocal relationship. Joint Bone Spine 86(5):576-582

11. Kushiyama A, Nakatsu Y, Matsunaga Y, Yamamotoya T, Mori K, Ueda K et al (2016) Role of uric acid metabolism-related inflammation in the pathogenesis of metabolic syndrome components such as atherosclerosis and nonalcoholic steatohepatitis. Mediators Inflam 2016:8603164

12. El Ridi R, Tallima H (2017) Physiological functions and pathogenic potential of uric acid: a review. J Adv Res 8(5):487-493

13. Kapoor M, Martel-Pelletier J, Lajeunesse D, Pelletier JP, Fahmi H (2011) Role of proinflammatory cytokines in the pathophysiology of osteoarthritis. Nat Rev Rheumatol 7:33-42

14. Busso N, So A (2010) Mechanisms of inflammation in gout. Arthritis Res Ther 12(2):206

15. Yokose C, Chen M, Berhanu A, Pillinger MH, Krasnokutsky S (2016) Gout and osteoarthritis: associations, pathophysiology, and therapeutic implications. Curr Rheumatol Rep 18(10):65

16. Denoble AE, Huffman KM, Stabler TV, Kelly SJ, Hershfield MS, McDaniel GE et al (2011) Uric acid is a danger signal of increasing risk for osteoarthritis through inflammasome activation. Proc Natl Acad Sci U S A 108(5):2088-2093

17. Xiao L, Lin S, Zhan F (2019) The association between serum uric acid level and changes of MRI findings in knee osteoarthritis: a retrospective study (A STROBE-compliant article). Medicine 98(21):e15819

18. Kim SK, Kwak SG, Choe JY (2018) Serum uric acid level is not associated with osteoarthritis in Korean population: data from the Seventh Korea National Health and Nutrition Examination Survey 2016. Rheumatol Int 38(11):2077-2085 
19. Altman R, Asch E, Bloch D, Bole G, Borenstein D, Brandt K et al (1986) Development of criteria for the classification and reporting of osteoarthritis: classification of osteoarthritis of the knee. Arthritis Rheum 29(8):1039-1049

20. Krasnokutsky S, Oshinsky C, Attur M, Ma S, Zhou H, Zheng F et al (2017) Serum urate levels predict joint space narrowing in non-gout patients with medial knee osteoarthritis. Arthritis Rheumatol 69(6):1213-1220

21. Hunter DJ, Altman RD, Cicuttini F, Crema MD, Duryea J, Eckstein F et al (2015) OARSI clinical trials recommendations: knee imaging in clinical trials in osteoarthritis. Osteoarthritis Cartilage 23:698-715

22. Bellamy N, Buchanan WW, Goldsmith CH, Campbell J, Stitt LW (1988) Validation study of WOMAC: a health status instrument for measuring clinically important patient relevant outcomes to antirheumatic drug therapy in patients with osteoarthritis of the hip or knee. J Rheumatol 15: 1833-1840

23. Maiuolo J, Oppedisano F, Gratteri S, Muscoli C, Mollace V (2016) Regulation of uric acid metabolism and excretion. Int J Cardiol 213:8-14

24. Kellgren JH, Lawrence JS (1957) Radiological assessment of osteoarthrosis. Ann Rheum Dis 16:494-502

25. Dupuis DE, Beynnon BD, Richard MJ, Novotny JE, Skelly JM, Cooper SM (2003) Precision and accuracy of joint space width measurements of the medial compartment of the knee using standardized MTP semi-flexed radiographs. Osteoarthritis Cartilage 11(10):716-724

26. Srivastava SR, Srivastava RN, Sharma AC, Raj S (2018) Serum uric acid levels influence osteoarthritis knee in non-gout population: does reference range need a revisit? Osteoarthritis Cartilage 26(1):S60-S474

27. Ding X, Zeng C, Wei J, Li H, Yang T, Zhang Y, Xiong YL et al (2016) The associations of serum uric acid level and hyperuricemia with knee osteoarthritis. Rheumatol Int 36(4):567-573

28. Sun Y, Brenner H, Sauerland S, Gunther KP, Puhl W, Sturmer T (2000) Serum uric acid and patterns of radiographic osteoarthritis - the UIm Osteoarthritis Study. Scand J Rheumatol 29(6):380-386

29. Howard RG, Samuels J, Gyftopoulos S, Krasnokutsky S, Leung J, Swearingen $C L$ et al (2015) Presence of gout is associated with increased prevalence and severity of knee osteoarthritis among older men: results of a pilot study. J Clin Rheumatol 21(2):63-71

30. Das SK, Ramakrishnan S, Mishra K, Srivastava R, Agarwal GG, Singh R et al (2002) A randomized controlled trial to evaluate the slow-acting symptommodifying effects of colchicine in osteoarthritis of the knee: a preliminary report. Arthritis Rheum 47(3):280-284

31. Leung YY, Haaland B, Huebner JL, Wong SBS, Tjai M, Wang C et al (2018) Colchicine lack of effectiveness in symptom and inflammation modification in knee osteoarthritis (COLKOA): a randomized controlled trial. Osteoarthritis Cartilage 26(5):631-640

32. Anderson JJ, Felson DT (1988) Factors associated with osteoarthritis of the knee in the first National Health and Nutrition Examination Survey (NHANES I). Evidence for an association with overweight, race, and physical demands of work. Am J Epidemiol 128(1):179-189

33. Bagge E, Bjelle A, Eden S, Svanborg A (1991) Factors associated with radiographic osteoarthritis: results from the population study 70-year-old people in Goteborg. J Rheumatol 18(8):1218-1222

34. Richmond RS, Carlson CS, Register TC, Shanker G, Loeser RF (2000) Functiona estrogen receptors in adult articular cartilage: estrogen replacement therapy increases chondrocyte synthesis of proteoglycans and insulin-like growth factor binding protein 2. Arthritis Rheum 43(9):2081-2090

35. Koga M, Saito H, Mukai M, Kasayama S, Yamamoto T (2009) Factors contributing to increased serum urate in postmenopausal Japanese females. Climacteric 12(2):146-152

36. Haugen IK, Slatkowsky-Christensen B, Boyesen P, Sesseng S, van der Heijde D, Kvien TK (2016) MRI findings predict radiographic progression and development of erosions in hand osteoarthritis. Ann Rheum Dis 75(1):117-123

37. Roemer FW, Kwoh CK, Hannon MJ, Hunter DJ, Eckstein F, Fujii T et al (2015) What comes first? Multitissue involvement leading to radiographic osteoarthritis: magnetic resonance imaging-based trajectory analysis over four years in the osteoarthritis initiative. Arthritis Rheumatol 67(8):2085-2096

38. Felson DT, Niu J, Neogi T, Goggins J, Nevitt MC, Roemer F et al (2016) Synovitis and the risk of knee osteoarthritis: the MOST Study. Osteoarthritis Cartilage 24(3):458-464

39. Krasnokutsky S, Belitskaya-Lévy I, Bencardino J, Samuels J, Attur M, Regatte R et al (2011) Quantitative magnetic resonance imaging evidence of synovial proliferation is associated with radiographic severity of knee osteoarthritis. Arthritis Rheum 63(10):2983-2991
40. McQueen FM, Chhana A, Dalbeth N (2012) Mechanisms of joint damage in gout: evidence from cellular and imaging studies. Nat Rev Rheumatol 8(3): 173-181

41. Shi $Y$ (2010) Caught red-handed: uric acid is an agent of inflammation. J Clin Invest 120(6):1809-1811

\section{Publisher's Note}

Springer Nature remains neutral with regard to jurisdictional claims in published maps and institutional affiliations.

\section{Submit your manuscript to a SpringerOpen ${ }^{\circ}$ journal and benefit from:}

- Convenient online submission

- Rigorous peer review

- Open access: articles freely available online

- High visibility within the field

- Retaining the copyright to your article

Submit your next manuscript at $\boldsymbol{\nabla}$ springeropen.com 